\title{
Thermomechanical fatigue in single crystal superalloys
}

\author{
Johan J. Moverare ${ }^{1, a}$ and Roger C. Reed ${ }^{2}$ \\ ${ }^{1}$ Engineering Materials, Dept. of Management and Engineering, Linköping University, 58183 Linköping, Sweden \\ ${ }^{2}$ Department of Engineering Science, University of Oxford, Parks Road, Oxford OX1 3PJ, UK
}

\begin{abstract}
Thermomechanical fatigue (TMF) is a mechanism of deformation which is growing in importance due to the efficiency of modern cooling systems and the manner in which turbines and associated turbomachinery are now being operated. Unfortunately, at the present time, relatively little research has been carried out particularly on TMF of single crystal (SX) superalloys, probably because the testing is significantly more challenging than the more standard creep and low cycle fatigue (LCF) cases; the scarcity and relative expense of the material are additional factors. In this paper, the authors summarise their experiences on the TMF testing of SX superalloys, built up over several years. Emphasis is placed upon describing: (i) the nature of the testing method, the challenges involved in ensuring that an given testing methodology is representative of engine conditions (ii) the behaviour of a typical Re-containing second generation alloy such as CMSX-4, and its differing performance in out-of-phase/in-phase loading and crystallographic orientation and (iii) the differences in behaviour displayed by the Re-containing alloys and new Re-free variants such as STAL15. It is demonstrated that the Re-containing superalloys are prone to different degradation mechanisms involving for example microtwinning, TCP precipitation and recrystallisation. The performance of STAL15 is not too inferior to alloys such as CMSX-4, suggesting that creep resistance itself does not correlate strongly with resistance to TMF. The implications for alloy design efforts are discussed.
\end{abstract}

\section{Introduction}

Nickel-based superalloys are designed to withstand extreme conditions of temperature and loading during operation. Their performance in critical hot gas path components such as vanes, blades and combustors largely limits the durability of modern gas turbines. Such components experience complex thermal and mechanical loading, especially during engine start-up and shut down, when the thermal gradients in conjunction with mechanical constraints and the time variation of both the temperature and the stresses eventually may lead to fatigue damage, a phenomenon often referred to as thermomechanical fatigue (TMF). Broadly speaking, TMF failure is promoted when plastic strains cannot be accommodated at low temperatures and creep deformation in combination with oxidation occurs at high temperature. However, microstructural stability plays an important role and it is now established that there are significant interactions between the degradation mechanisms occurring at high and low temperatures. In addition the effect of coatings must be considered.

Although material for critical hot components has traditionally been evaluated with respect to creep, the deformation characteristics under TMF conditions are now just as important and need to be understood and quantified if component lifetime estimates are to be accurate. Indeed, one of the major causes of failure of hot gas path

\footnotetext{
${ }^{a}$ Corresponding author: johan.moverare@liu.se
}

components in current gas turbines is thermally induced cracking aggravated by oxidation. The main load cycle of such components will always be of the type start-up, operation and shut down, but the length and detailed form of such a load cycle will depend on the turbine application: i.e. whether it is a jet engine used for aircraft propulsion, or alternatively a stationary gas turbine situated in a powerplant. The detrimental effect of a load cycle can therefore vary significantly between different types of operating conditions.

Thermomechanical fatigue in superalloys is growing in importance since gas turbines are being operated under ever more arduous conditions, to reduce fuel usage and $\mathrm{CO}_{2}$ emissions and to maximise performance. The introduction of thermal barrier coatings (TBCs) and sophisticated and more efficient cooling systems together with higher operating temperatures have lead to a tendency for thinner wall thicknesses which means that the thermal gradients are steeper. In addition, the changes in demands and competition within the power generation market forces the power plants in many countries to operate under cyclic conditions. Today, it is common to have gas turbines in intermittent operation in order to balance irregular fluctuations of the peak load requirements of a grid. This means that the turbines either start and stop on a daily basis or have significant variations in power output over a very short period of time. This exacerbates the importance of the thermomechanical fatigue (TMF) effect. 


\section{TMF testing methodology}

Constraint of free thermal expansion and contraction is an intrinsic ingredient of the thermally induced fatigue process. It is therefore this which is of interest to reproduce on a laboratory scale. This can be achieved by means of thermal fatigue (TF) tests on specimens with internal constraints or by thermomechanical fatigue (TMF) tests on specimens with external constraints. TMF testing on specimens with external constraints was introduced in the early 70's; the pioneering work on superalloys was conducted at Pratt \& Whitney Aircraft on both smooth specimens and specimens suitable for studying TMF crack propagation $[1,2]$. However, testing of materials in TMF is still the subject of considerable debate, and standards for it are relatively new.

Most TMF tests are conducted in strain control using servo-electric or servo-hydraulic testing machines in which the stress and strains are measured using a load cell and high temperature extensometry, as in a normal LCF test at elevated temperature. Several methods such as direct and indirect induction heating, resistive heating as well as radiation heating have been successfully used for nonisothermal TMF testing, with direct induction heating being the most frequently employed [3].

In practice, several aspects require careful consideration if TMF testing is to be meaningful and accurate. In the mechanical strain-controlled TMF tests, the measurement and control of temperature is considered to be a most critical issue; this is because an error in the temperature gives rise to an error in the mechanical strain, which will in turn affect the stress response and the cyclic life measured. Thus, the temperature measurement was found to be the major origin of scatter in a recent round robin exercise [4]. Furthermore, as the specimen is continuously heated and cooled during TMF testing, thermal gradients are inevitable, and if not kept low enough these gradients will lead to premature failure of the test piece. Good control of the gradients both during cooling and heating is thus vital if testing conditions are to be appropriate. In TMF testing it is also, as for LCF-testing, important to reduce the misalignment in the load train to a minimum.

The fact that single crystal superalloys are highly anisotropic and also sometimes show a very localized deformation behaviour add an extra complexity to the testing of these materials. If deformation primarily occur along deformation bands that extend across the complete cross section of the specimen, as seen in Fig. 1, the interpretation of the results can be difficult if there also is a gradient in stress and temperature along the deformation band.

Apart from the difficulty to do the actual testing it is also of high importance to carefully select the test conditions to ensure that it is representative of real engine operation. This often requires very good knowledge of the component of interest with respect to stress, strain and temperature fields over the component and how these fields vary with time. In addition it is necessary to have good knowledge about the material behaviour in order to interpret the results; sometimes it is necessary to take the degradation effects that occur during long terms service into account $[5,6]$.

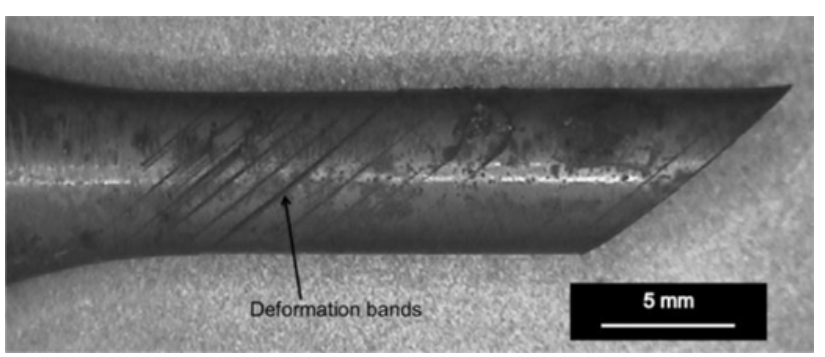

Figure 1. Deformation bands in STAL15 due to TMF.

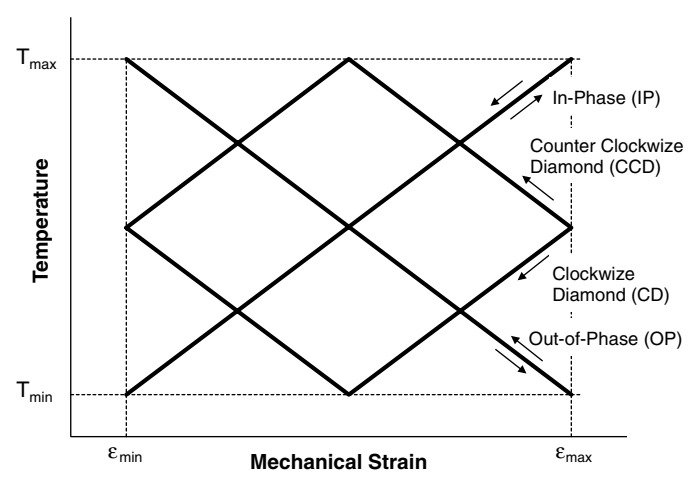

Figure 2. Illustration of different TMF-cycles.

TMF testing provides a powerful tool to assess the synergistic effects of combined thermal and mechanical loads and involves the cycling of the temperature $\mathrm{T}$ and mechanical strain $\varepsilon_{\text {mech }}$ with different phase shifts. The testing can thus be very component-specific and mimic more or less exactly the situation in a critical location of a component. However, in order to generate generic material data, it is common to stick to some simplified general cycles such as the in-phase (IP), the out-of-phase (OP) cycle or diamond cycles, see Fig. 2.

Focusing on the IP-cycle, we have a situation in which the tensile stress/strain loading coincides with the high-temperature part of the cycle. The deformation is thus characterized by high temperature creep in tension and low temperature plasticity in compression. For pure thermal loadings, this situation is found at "cold-spot areas" where the temperature is typically lower compared to the surrounding, e.g. near cooling holes or at structures inside cooling channels. However, the stresses/strains from thermal gradients can also be superimposed by external loads such as internal pressure and centrifugal forces which will further increase the tensile stress at elevated temperature. The combination of high tensile stresses at an elevated temperature and significant hold times can have a very detrimental effect on fatigue performance even for rather moderate temperatures.

The situation during an OP TMF-cycle is completely different. In this case the material undergoes creep relaxation in compression at high temperatures and plastic deformation in tension at low temperatures. This is the situation found at "hot-spot areas", i.e. small regions with higher temperature than the near environment which may occur on e.g. on a turbine blade airfoil or platform due to insufficient cooling. Since these areas will be in 


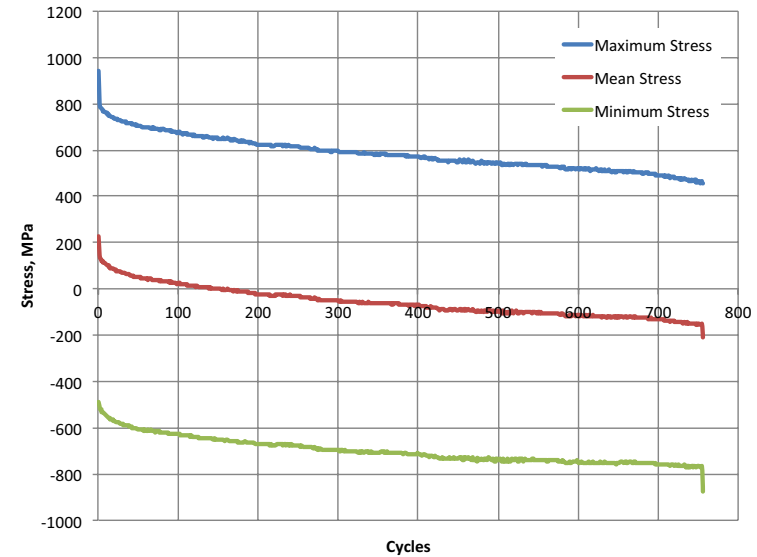

Figure 3. Evolution of maximum, mean and minimum stress during IP TMF testing $\left(100-750^{\circ} \mathrm{C}\right)$ of MD2.

compression during the high-temperature running period of the engine, high-temperature creep relaxation will cause the material to be in tension at shut-down. Since rafting and other degradation mechanisms occurring at high temperature are found to have a higher effect on the low-temperature properties than on the high-temperature ones, it can be readily concluded that the OP TMF-loading condition is quite different from an iso-thermal LCFloading.

In case of both IP and OP-TMF, the magnitude of the stresses in the cold end of the TMF cycle will increase with the amount of stress relaxation in the hot-end of the TMF cycle. Thus, the possibility for having reversed plasticity at engine shut-down will increase with the amount of stress relaxation during service. Thus the length of the time spent at high temperature will (apart from other degradation mechanisms) be important for the TMF life of the component. As creep always is present when engineering components are subjected to TMF conditions, it is strongly recommended also to introduce a dwell period at the maximum temperature in the TMF-test. The length of the dwell period often varies from a couple of seconds to several hours, but for practical reasons the dwell time in laboratory tests is often much shorter than the typical time of operation for the component of interest. This is especially the case for hot components in industrial turbines where the average time between start and stop can be up to 500 hours.

From a testing perspective, it is of course problematic to use very extended dwell times. Short dwell times are however also problematic when evaluating the results since the time dependent creep relaxation in one end of the TMF cycle can shift the mean stress significantly cycle by cycle during the test, as shown in Fig. 3. As a consequence no stabilized stress values will be achieved. For single crystal materials this is typically a problem for lower values of $\mathrm{T}_{\max }$ and short dwell times. One way to partly overcome this problem is to use a significantly longer dwell time in the first cycle. The industrial standard for some companies is to apply a 20 hour dwell time in the first cycle and 5 minutes dwell time in the following cycles [5]. Also the $\mathrm{R}$-value has to be selected carefully. By assuming that the minimum temperature is the reference temperature where

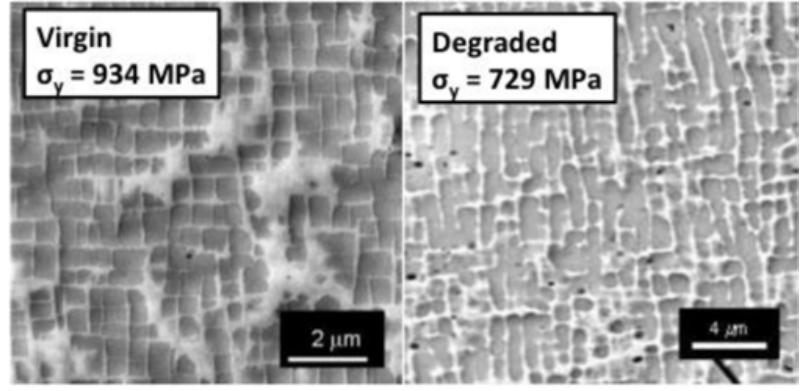

Figure 4. Microstructure of MD2 in virgin and degraded condition tensile tested at $20^{\circ} \mathrm{C}$ in the $\langle 001\rangle$ direction [9].

the mechanical strain is defined as "zero" the definition of the IP and OP cycles implies $R_{\varepsilon}=0$ for the IP cycle and $R_{\varepsilon}=-\infty$ for the OP cycle. Since both these R-values are non symmetric they will lead to a significant redistribution of stresses during the first cycle, i.e. the amount of plastic deformation is much larger in the first cycle compared to the following cycles. This partly contribute to the problem with the drift of mean stress as illustrated in Fig. 3 but this redistribution of stresses is the actual situation for most critical areas sensitive to fatigue. For single crystal superalloys, the plastic deformation in the first cycle is also crucial in order to enhance microstructural changes such as rafting. In fact rafting has been found to occur very rapidly during TMF testing [6]. Thus, to fully take the degradation effect into account it is often necessary to also do testing on service exposed material or to simulate service conditions by doing interrupted testing with an intermediate artificial long term ageing treatment [5].

Thus there are a number of testing parameters that have to be selected carefully in order to do TMF testing that is relevant from the component perspective. One parameter that is not included here but will be covered in the next section is the influence of the minimum temperature.

\section{Microstructural aspects of TMF}

\subsection{Influence of rafting \& minimum temperature}

The yield strength anomaly for the pure $\gamma^{\prime}$-phase $\left(\mathrm{Ni}_{3} \mathrm{Al}\right)$ is rather well known [7] but the implications of this phenomena on TMF testing of $\gamma-\gamma^{\prime}$ alloys and the influence of minimum temperature is often overseen. Both the $\gamma$ matrix and the $\gamma^{\prime}$-precipitate are relatively weak at room temperature. The remarkably high virgin material yield strength is a consequence of a favourable matrix/precipitate interaction associated with the carefully optimised, but thermodynamically unstable, virgin microstructure. Therefore it is only natural that a deviation from this carefully optimised microstructure will result in a reduction of the yield strength [8]. One example can be seen in Fig. 4, where the virgin microstructure with a room-temperature yield strength of $934 \mathrm{MPa}$ is compared to the microstructure of a degraded material with a room-temperature yield strength of $729 \mathrm{MPa}$. Even if the orientation and composition is identical there is a reduction of approximately $25 \%$ due to the degradation [9].

At higher temperatures, this class of alloys does not need to rely as much on a favourable matrix-particle 


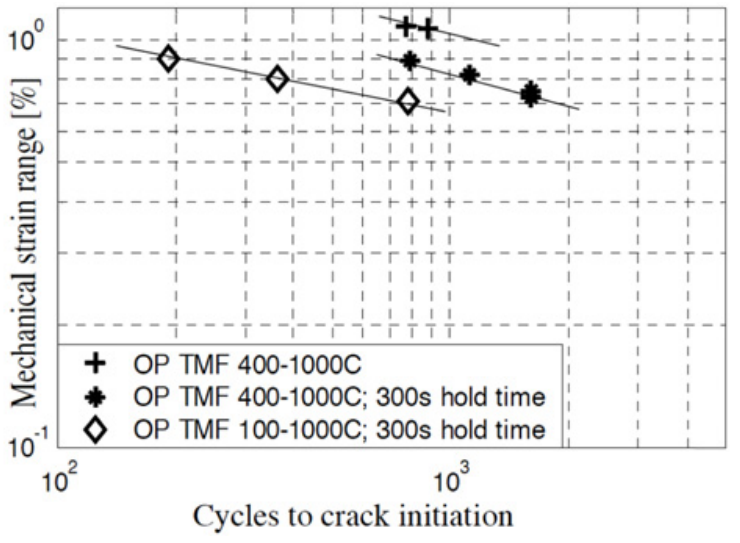

Figure 5. Influence of dwell and $\mathrm{T}_{\min }$ on the TMF crack initiation life of CMSX-4, from [8].

interaction, because the $\gamma^{\prime}$ imparts strength regardless of the shape and size of the particles. This is associated with the fact that the $\gamma^{\prime}$ shows an increasing yield strength from room temperature to about $700{ }^{\circ} \mathrm{C}$ [7]. Because rafting occurs during $\mathrm{TMF}$ tests with high $\mathrm{T}_{\max }$ and dwell times, it follows that the material will yield at a lower stress value than in the virgin condition but this effect will also increase with decreasing value of $\mathrm{T}_{\text {min }}$. When the material yields at a lower stress level, it will result in a higher inelastic strain range and a correspondingly reduced crack initiation life. This has been demonstrated for CMSX-4, see Fig. 5, where the TMF life was reduced by a factor of 3 when the $\mathrm{T}_{\min }$ was reduced from $400{ }^{\circ} \mathrm{C}$ to $100^{\circ} \mathrm{C}$ [8]. Similar behaviour has also been reported for directional solidified material [10].

\subsection{Slip and twinning}

During OP-TMF testing of second generation single crystals such as CMSX-4, highly localized twinning are found to be the main deformation mechanism [6,11-13]. In the study by Segersäll [14] it was found that for high strain ranges the twins often extend across the complete cross section of the specimen, which leads to a highly crystallographic fracture surface as seen in Figs. 6a and 6c. For lower strain ranges the fracture surface is more noncrystallographic and typically perpendicular to the fracture surface, as seen in Figs. 6b and 6d.

However, even for the non-crystallographic fracture surfaces in Figs. $6 \mathrm{~b}$ and $6 \mathrm{~d}$, twinning was found to be the main deformation mechanism. This is illustrated in Fig. 7 were it can be seen that the crack is surrounded by crystallographic deformation and twins. During OP TMF testing of specimens oriented along the $\langle 001\rangle$ direction, twinning will occur during compression at elevated temperature as a result of activity of the $\langle 112\rangle\{111\}$ type slip system which requires the passage of $\mathrm{a} / 3\langle 112\rangle$ dislocations through both the matrix and superlattice on adjacent $\{111\}$ planes [15].

In the same study by Segersäll [14] OP TMF testing was also performed on $\langle 011\rangle$ oriented samples and for this direction twinning was less frequently observed; especially at high strain ranges, deformation occurred in very localized deformation bands but without formation of

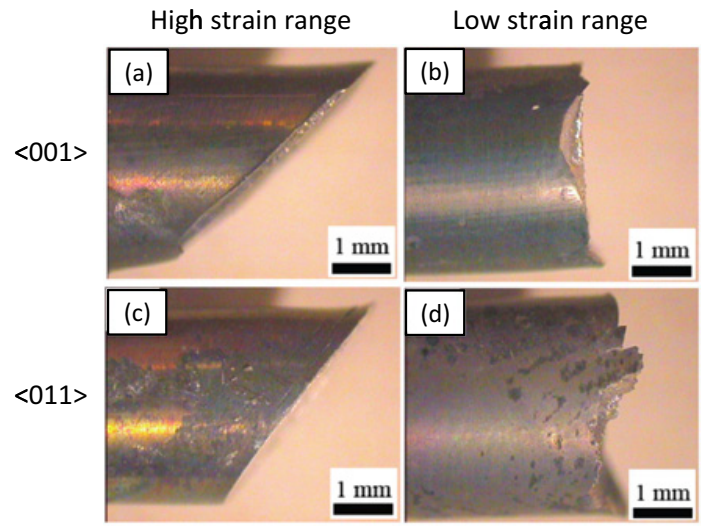

Figure 6. Influence of strain range on the crack appearance for CMSX-4 during OP-TMF 100-850 ${ }^{\circ} \mathrm{C}$ [14].

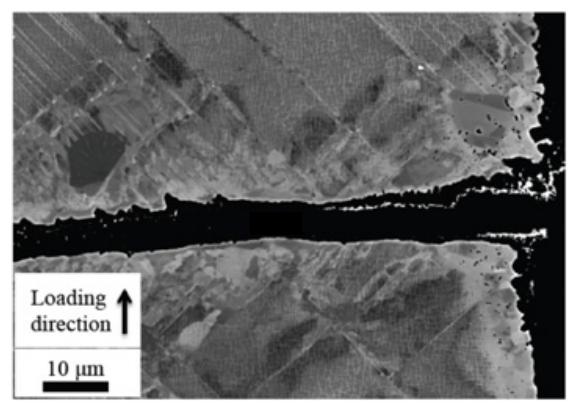

Figure 7. Crystallographic deformation and twins surrounding a secondary crack in CMSX-4 after OP-TMF $100-850^{\circ} \mathrm{C}$ testing (low strain range for the $\langle 001\rangle$-direction) [14].

twins. The deformation occur by slip and cutting of the $\gamma^{\prime}$ particles along the $\{111\}$ planes but seems to also be accompanied by a local rafting mechanism as illustrated in Fig. 8. The orientation of the single crystal relative to the applied load is thus important for the formation of deformation twins during TMF.

In addition to the influence of crystallographic orientation, single crystal superalloys are known to display a tension/compression asymmetry in both plasticity and creep $[9,16,17]$. Consequently, different deformation behaviour can be expected for IP and OP cycling during TMF. This has been illustrated in a recent study by Segersäll [18] where a clear difference between IP and OP TMF was found: for the $\langle 001\rangle$ direction twinning was much more pronounced during OP TMF while for the $\langle 011\rangle$ direction IP TMF leads to twinning.

While the observations described above are typical for second generation single crystal superalloys with a high volume fraction of $\gamma^{\prime}$ such as CMSX-4, a somewhat different behaviour is observed in Re-free single crystals with a lower fraction of $\gamma^{\prime}$, such as SCA425 and STAL15 (Ni-5Co-1Mo-3.7W-15Cr-4.55Al-8Ta). Even if twinning sometimes is observed, the main deformation mechanism is slip along the $\{111\}$ planes even during OP TMF testing along the $\langle 001\rangle$ direction $[19,20]$. Slip deformation is concentrated within a small number of bands along the specimen gauge length. The thickness of these bands can be up to $2 \mu \mathrm{m}$ and they often develop across the entire 


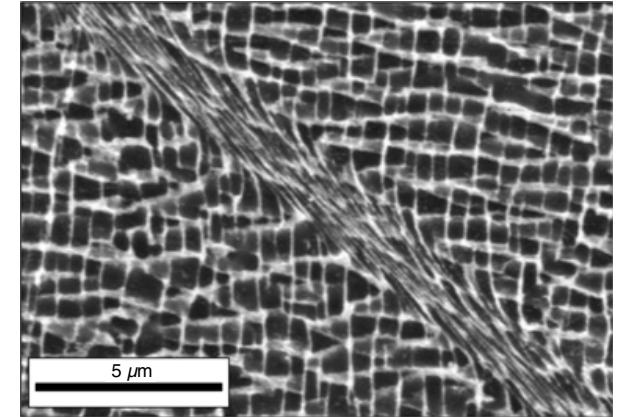

Figure 8. Deformation band formed in CMSX-4 during OP-TMF $\left(100-850^{\circ} \mathrm{C}\right)$ testing along the $\langle 011\rangle$ direction [14]. Stress axis is the vertical direction.

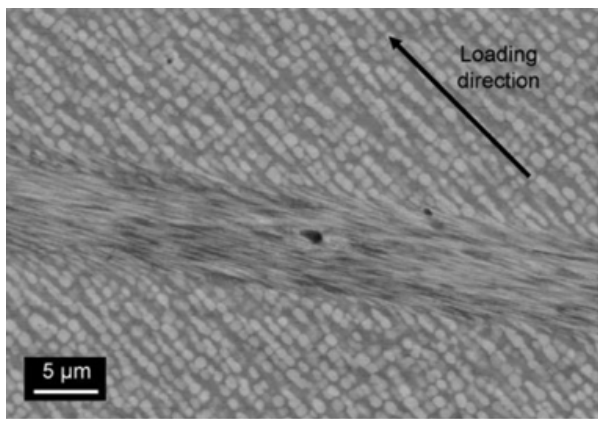

Figure 9. Deformation band formed in SCA425Hf during OPTMF $\left(100-950^{\circ} \mathrm{C}\right)$ testing along the $\langle 001\rangle$ direction [19].

cross-section of the specimen. The structure inside the bands can be seen in Fig. 9.

While during creep, dislocation activity is mainly restricted to the $\gamma$ matrix phase, TMF failure is promoted by localized shear banding with the $\gamma^{\prime}$ phase penetrated by dislocations. Consistently it was found in [20] that the size of the $\gamma^{\prime}$-precipitates influences creep and TMF differently. The TMF resistance increased with increasing primary $\gamma^{\prime}$ size while in creep the finer primary $\gamma^{\prime}$ microstructure gave better performance. In addition it has also been found that $\mathrm{Si}$ which can be believed to strengthen the $\gamma^{\prime}$-phase significantly improves the resistance to TMF failure [11].

\subsection{TCP formation and recrystallization}

The plastic and elastic energy stored inside the deformation bands described in the previous section can be expected to be rather high. This will explain their susceptibility to TCP formation and recrystallization. In reference [6] it was found that within the deformation bands in CMSX-4 after OP TMF testing with a maximum temperature of $1000{ }^{\circ} \mathrm{C}$, small precipitates of topologically close-packed (TCP) phases could be observed, see Fig. 10. WDS analysis revealed that the precipitates are rich in Ta, W and Re, consistent with CMSX-4's known propensity for precipitation of the $\mu$-phase. Since the TCP phases were observed only inside or at the twin/matrix interface, the precipitation must have occurred after the twinning. Thus the precipitation of the TCP phases must be promoted by the plastic and elastic energy associated by the deformation band. In the case of twinning there might
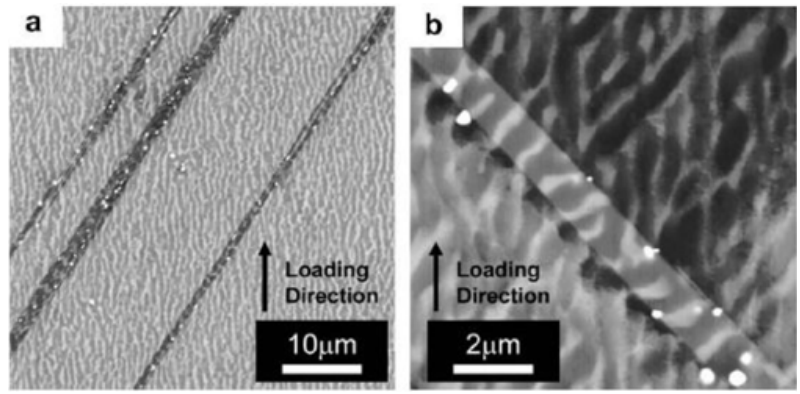

Figure 10. Deformation band with TCP phases formed in CMSX-4 during OP-TMF $\left(100-1000^{\circ} \mathrm{C}\right)$ testing along the $\langle 001\rangle$ direction [6].

also be the possibility that thermally-activated diffusional processes that must occur during the formation of the twin can lead to segregation of heavy elements [21,22] which also promotes the formation of TCP-phases.

When long term ageing at $1000^{\circ} \mathrm{C}$ for 4000 hours was performed on CMSX-4 a lot of needle-shaped precipitates were found, which act as obstacles for the slip or twin bands. As a consequence, the slip and twin bands cannot extend across the complete cross-section of the specimen and thus prevent the deformation from being as highly localized as for the virgin material. In order to accommodate the plastic deformation in the aged material, more slip or twin bands are activated compared to virgin material, giving a more dispersed deformation behaviour [6].

In addition to TCP formation it has frequently been observed that recrystallization eventually will occur within the bands of localized deformation. The absence of grain boundary strengthening elements in single crystal materials leads to easy crack propagation along the recrystallized bands. The recrystallization is illustrated in Fig. 11 for an OP TMF test on CMSX-4 with a maximum temperature of $1000{ }^{\circ} \mathrm{C}$ [6]. Similar observations have also been found in TMF tests with a maximum temperature of $850^{\circ} \mathrm{C}$ [14] and in MD2 even in test with $750{ }^{\circ} \mathrm{C}$ as the maximum temperature [18].

The starting point for the recrystallization process has been found to be the intersection of twins growing in different directions. Once recrystallization has started, it can develop further along the twin directions [6]. Interestingly, no recrystallization is observed for CMSX-4 in the long term aged condition [6] due to the smaller width and length of the twins and the more dispersed deformation behaviour.

\section{Conclusions}

- During TMF testing if the highest temperature is above $900^{\circ} \mathrm{C}$, rafting occurs rather rapidly. Consequently, the low temperature yield strength is reduced and there is a strong influence on the TMF life when the minimum temperature is altered from $400{ }^{\circ} \mathrm{C}$ to $100^{\circ} \mathrm{C}$.

- Twinning is an important deformation mechanism during TMF in single crystal superalloys. Twinning is typically more pronounced during OP TMF 


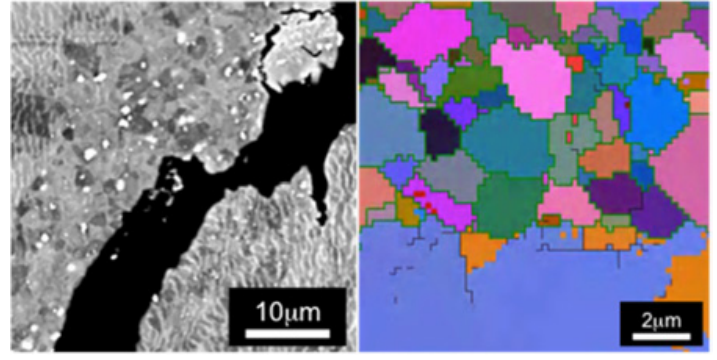

Figure 11. Left: micrograph showing TCP formation and recrystallization near a crack formed during OP TMF (100$950^{\circ} \mathrm{C}$ ) testing of CMSX-4. Right: region within a band of localized deformation characterized by EBSD, confirming recrystallization [6].

testing along the $\langle 001\rangle$ direction and during IP TMF testing along the $\langle 011\rangle$ direction.

- In alloys with a lower fraction of $\gamma^{\prime}$, twinning is less frequently observed but still the deformation is very localized to a few deformation bands.

- Deformation bands formed by TMF are susceptible to TCP formation and recrystallization. Recrystallization is particularly detrimental to TMF resistance and crack formation occurs readily along the recrystallized deformation bands.

- When recrystallization occurs in this way, the nucleation sites are often the intersection of two propagating twins of different orientations. During OP-TMF testing recrystallization has been observed in tests with a maximum temperatures as low as $750{ }^{\circ} \mathrm{C}$.

The work has been financially supported by Siemens Industrial Turbomachinery $\mathrm{AB}$ in Finspång, Sweden and the Swedish Energy Agency, via the Research Consortium of Materials Technology for Thermal Energy Processes, Grant No. KME-502.

\section{References}

[1] Hopkins S. Testing, Low-Cycle Thermal Mechanical Fatigue, in: ASTM STP 612. 1976

[2] Rau Jr C, Gemma A, Leverant G. ThermalMechanical Fatigue Crack Propagation in Nickeland Cobalt-Base Superalloys Under Various StrainTemperature Cycles, in: ASTM STP 520. 1972
[3] Hahner P, Rinaldi C, Bicego V, Affeldt E, Brendel $\mathrm{T}$, Andersson H, Beck T, Klingelhoffer H, Kuhn H, Koster A. Int J Fatigue 2008;30:372

[4] Loveday M, Bicego V, Hahner P, Klingelhoffer H, Kuhn H, Roebuck B. Int J Fatigue 2008;30:382

[5] Hasselqvist M, Moverare J. Vol 5 Turbo Expo 2007 2007; 1:131

[6] Moverare J, Johansson S, Reed RC. Acta Mater 2009;57:2266

[7] Ezz SS, Pope DP, Paidar V. Acta Metall 1982;30:921

[8] Arrell D, Hasselqvist M, Sommer J, Moverare J. On TMF Damage, Degradation Effects, and the Associated Tmin Influence on TMF Test Results in $\gamma / \gamma$ ' Alloys, in: Superalloys 2004. 2004

[9] Leidermark D, Moverare J, Johansson S, Simonsson K, Sjöström S. Acta Mater 2010;58:4986

[10] Kupkovits R a., Smith DJ, Neu RW. Procedia Eng 2010;2:687

[11] Moverare JJ, Segersäll M, Sato A, Johansson S, Reed RC. Thermomechanical Fatigue of SingleCrystal Superalloys: Influence of Composition and Microstructure, in: Superalloys 2012

[12] Zhang JX, Ro Y, Zhou H, Harada H. Scr Mater 2006;54:655

[13] Zhang JX, Harada H, Ro Y, Koizumi Y, Kobayashi T. Acta Mater 2008;56:2975

[14] Segersäll M, Moverare JJ, Simonsson K, Johansson S. Deformation and Damage Mechanisms During Thermomechanical Fatigue of a Single-Crystal Superalloy in the $\langle 001\rangle$ and $\langle 011\rangle$ Directions, in: Superalloys 2012

[15] Knowles DM, Gunturi S. Mater Sci Eng A 2002;328:223

[16] Heredia FE, Pope DP. Acta Metall 1986;34:279

[17] Segersäll M, Moverare JJ, Leidermark D, Simonsson K. Metall Mater Trans A 2014; Accepted:10.1007/s11661

[18] Segersäll M, Moverare JJ, Leidermark D, Johansson S. In- and out-of-phase Thermomechanical Fatigue of a Ni-Based Single-Crystal Superalloy, in: EuroSuperalloys 2014

[19] Moverare JJ, Johansson S. Mater Sci Eng A 2010;527:553

[20] Sato A, Moverare J, Hasselqvist M, Reed RC. Metall Mater Trans A 2012;43:2302

[21] Kovarik L, Unocic RR, Li J, Mills MJ. JOM 2009;61:42

[22] Kovarik L, Unocic RR, Li J, Sarosi P, Shen C, Wang Y, Mills MJ. Prog Mater Sci 2009;54:839 